\title{
Characterisation of a plastic scintillation detector to be used in a multicentre stereotactic radiosurgery dosimetry audit
}

\author{
A. Dimitriadis ${ }^{\mathrm{a}, \mathrm{b}, *}$, I. Silvestre Patallo ${ }^{\mathrm{b}}$, I. Billas ${ }^{\mathrm{b}}, \mathrm{S}$. Duane ${ }^{\mathrm{b}}$, A. Nisbet ${ }^{\mathrm{a}, \mathrm{c}}$, C.H. Clark ${ }^{\mathrm{b}, \mathrm{c}}$ \\ a Department of Physics, University of Surrey, Guildford, Surrey, UK \\ b Radiation Dosimetry Group, National Physical Laboratory, Teddington, UK \\ ${ }^{\mathrm{c}}$ Medical Physics Department, Royal Surrey County Hospital, Guildford, UK
}

\section{A R T I C L E I N F O}

\section{Keywords:}

Scintillation detector

Dosimetry

Radiotherapy

Radiosurgery

Audit

\begin{abstract}
A B S T R A C T
Scintillation detectors are considered highly suitable for dosimetric measurement of small fields in radiotherapy due to their near-tissue equivalence and their small size. A commercially available scintillation detector, the Exradin W1 (Standard Imaging, Middleton, USA), has been previously characterised by two independent studies (Beierholm et al., 2014; Carrasco et al., 2015a, 2015b) but the results from these publications differed in some aspects (e.g. energy dependence, long term stability). The respective authors highlighted the need for more studies to be published (Beierholm et al., 2015; Carrasco et al., 2015a, 2015b).

In this work, the Exradin W1 was characterised in terms of dose response, dependence on dose rate, energy, temperature and angle of irradiation, and long-term stability. The observed dose linearity, short-term repeatability and temperature dependence were in good agreement with previously published data. Appropriate corrections should therefore be applied, where possible, in order to achieve measurements with low-uncertainty. The angular dependence was characterised along both the symmetrical and polar axis of the detector for the first time in this work and a dose variation of up to $1 \%$ was observed. The response of the detector was observed to decrease at a rate of approximately $1.6 \% \mathrm{kGy}^{-1}$ for the first $5 \mathrm{kGy}$ delivered, and then stabilised to $0.2 \% \mathrm{kGy}^{-1}$ in the subsequent $20 \mathrm{kGy}$.

The main goal of this work was to assess the suitability of the Exradin W1 for use in dose verification measurements for stereotactic radiosurgery. The results obtained confirm that the detector is suitable for use in such situations. The detector is now utilised in a multi-centre stereotactic radiosurgery dosimetric audit, with the application of appropriate correction factors.
\end{abstract}

\section{Introduction}

Plastic scintillation detectors (PSD) have been investigated for their performance in medical radiation dosimetry for more than two decades (Beaulieu et al., 2013). They are considered suitable for radiotherapy applications, as they can be manufactured in small sizes, have tissue equivalent density and are capable of performing real-time measurements. As far as the authors are aware, there is currently only one commercially available PSD for small field photon dosimetry applications, the Exradin W1 (Standard Imaging, Middleton, WI, USA).

The Exradin W1 became commercially available in the UK in the summer of 2014. The sensitive volume of the detector is a $3 \mathrm{~mm}$ (length) by $1 \mathrm{~mm}$ (diameter) polystyrene cylinder, doped with scintillating agents. It is encased within an opaque enclosure made of epoxy resin and acrylonitrile butadiene styrene (ABS). The scintillator is coupled to an optical fibre with a $1 \mathrm{~mm}$ diameter polymethyl methacrylate (PMMA) core and a $2.2 \mathrm{~mm}$ diameter polyethylene jacket. The scintillator-fibre coupling is externally protected with a polyimide sheath. The fibre is $3 \mathrm{~m}$ long and is attached to a photodiode box. Although the manufacturer does not provide a detailed description of the contents of the photodiode box, it is understood to include instrumentation for the chromatic separation of light (dichroic filters and photodiodes). The other end of the photodiode box has two connectors for transmitting the electrical charges collected to a dual channel electrometer. Alternatively, two individual electrometers may be used, as long as they are able to detect the small charges produced by the PSD, which are in the pico-Coulomb range. Measurements with the PSD in two setup orientations, of maximum and minimum optical fibre in the radiation field, are used for the determination of the Cherenkov Light Ratio (CLR) correction. This is necessary to remove the stem signal produced by irradiation of the fibre. The manufacturer provides a $30 \times 30 \mathrm{~cm}^{2}$ polystyrene calibration slab that allows placement of the detector fibre in the minimum and maximum fibre orientations (Fig. 1).

* Corresponding author at: Department of Physics, University of Surrey, Guildford, Surrey, UK. 


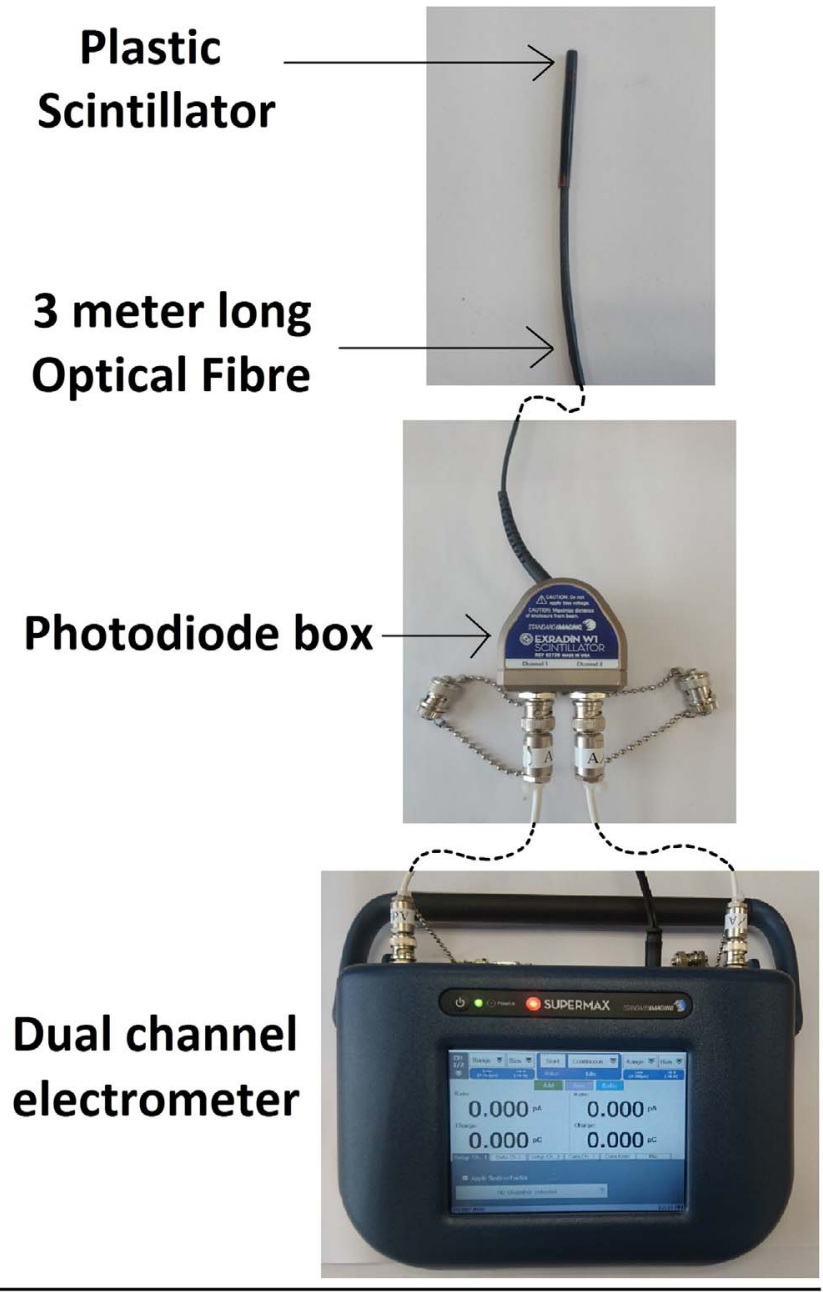

\section{$30 \times 30 \mathrm{~cm}$ Calibration Slab}



Minimum fibre



Maximum fibre
Fig. 1. Components of the Exradin W1 plastic scintillation detector, SuperMAX electrometer and calibration slab.

A previous study performed Monte Carlo simulations of different detectors in small fields, reported that the Exradin W1's response in small fields was expected to be within $1 \%$ of simulated output factors (Kamio and Bouchard, 2014). Similar findings were experimentally validated by another study in the measurement of small field output factors (Underwood et al., 2013) and the PSD was subsequently used to determine correction factors for other small field detectors. A third independent study, utilised a similar methodology and produced comparable results, further validating further the suitability of this detector for use in small fields (Silvestre et al., 2016). The detector was also used in an Italian multi-centre study for the measurement of output factors, which also confirmed its suitability for small fields
(Pasquino et al., 2016). Although the available evidence supports the use of this detector in small fields, the dosimetric characteristics of the detector need to be further investigated to assess its suitability for patient-specific small field dose verification, such as used in clinical Stereotactic Radiosurgery (SRS).

The Exradin W1 has recently been recently characterised by two independent and almost simultaneously published studies (Beierholm et al., 2014; Carrasco et al., 2015a, 2015b). The results demonstrate promising dosimetric characteristics. However, slightly different results were observed for the energy dependence and the long term stability of the detector, which were highlighted and discussed in a letter to the editor (Beierholm et al., 2015). A response to this letter commented on the possibility that such differences may be inherent to the different detectors and highlighted the need for more studies investigating the dosimetric properties of the Exradin W1 (Carrasco et al., 2015a, 2015b).

There are limited published studies on the use of PSDs for dose verification purposes (Klein et al., 2012; Ottosson et al., 2015), although this limited evidence does not suggest their applicability in this area. In order to determine whether the Exradin W1 is suitable for SRS dose verification some additional investigations needed to be performed. These include the angular dependence of the detector along its polar axis and assessment of the manual collection mode for high dose measurements, which have not been previously been published.

The purpose of this study was therefore to conduct a full dosimetric characterisation of the Exradin W1 in order to verify previously published results, further investigate dosimetric characteristics where different results have been published, and extend the dosimetric characterisation with the aim of verifying the Exradin W1's suitability for use in the methodology of a national SRS dosimetry audit (Dimitriadis et al., 2016a).

\section{Materials and methods}

The W1 detector was connected to a SuperMAX dual channel (standard Imaging, Middleton, WI, USA). The readings from both channels were acquired in the low range (pC), using both triggered and manual collection modes. All factors and dose measurements were calculated manually using the spectral method (Guillot et al., 2011). Channel 1 of the electrometer collected signal produced mainly from the scintillator, and channel 2 collected signal mainly produced from Cherenkov in the stem. When trigger mode collection was used, channel 1 was automatically initiated and ended the measurement using the default threshold values of $0.4 \mathrm{pA}$ (start) and $0.2 \mathrm{pA}$ (stop) respectively. Manual collections were acquired by starting the collection immediately before the beam came on and stopping the collection after the beam went off and the dose-rate indications for both channels of the electrometer returned to zero. Leakage currents were occasionally noticed during the experiments. In order to minimise these, the detector was left to equilibrate for at least $10 \mathrm{~min}$. It was then preirradiated with a dose of approximately $10 \mathrm{~Gy}$ and the electrometer was then corrected for background leakage. The photodiode box was positioned as from the primary beam as possible and shielded from scattered radiation, as there is evidence to suggest that similar instrumentation is susceptible to noise from scattered radiation (Liu et al., 2012). The irradiations were performed with a nominal $6 \mathrm{MV}$, $10 \mathrm{MV}$ and $15 \mathrm{MV}$ photon beam from an Elekta Versa HD linear accelerator (linac) and a Theratron ${ }^{60}$ Cobalt unit.

The work reported here was undertaken with the PSD positioned in both perpendicular and parallel orientations to the radiation beam. For perpendicular irradiations, the detector was calibrated in its calibration slab (Fig. 1), using sufficient $30 \times 30 \mathrm{~cm}$ blocks of water equivalent plastic material (WT1) to ensure full build-up and backscatter. The detector was placed at the radiation isocentre at $5 \mathrm{~cm}$ depth in WT1, $95 \mathrm{~cm}$ Source-to-Surface Distance (SSD), and with $15 \mathrm{~cm}$ WT1 for backscatter. A $40 \times 40 \mathrm{~cm}$ field size was employed. Absolute dose 
measurements were also performed under isocentric conditions in a $10 \times 10 \mathrm{~cm}$ field, using ionisation chambers with calibration traceable to the primary standard held at the National Physical Laboratory (NPL, Teddington, UK). For parallel irradiations, the PSD was placed in water, in a Blue Phantom plotting tank (IBA, Belgium). The PSD manufacturer's recommended calibration procedure was followed for this detector orientation (Morin et al., 2013). Variations in the setup conditions from those stated above were used for some tests. These are described in the relevant sections that follow.

\subsection{Dose response, collection mode and short-term repeatability}

The response of the PSD to dose was measured in ${ }^{60} \mathrm{Co}, 6 \mathrm{MV}$, $10 \mathrm{MV}$ and $15 \mathrm{MV}$ photon beams with respective $\mathrm{TPR}_{20 / 10 \mathrm{~cm}}$ Quality Indices (QI) of $0.682,0.733$ and 0.758 for the latter three beam energies. For the linac beams, a PTW Semiflex ionisation chamber was placed $5 \mathrm{~cm}$ below the PSD to account for any variations in output. Measurements were performed using both triggered and manual collection modes in order to investigate their differences. The dose range investigated was from 0.1 to $40 \mathrm{~Gy}$. The short-term repeatability of the detector was also assessed by performing repeated measurements in the same conditions in a ${ }^{60} \mathrm{Co}$ beam.

\subsection{Dose-rate and dose-per-pulse}

The dose-rate dependence was investigated in air using a wooden clamp to ensure low unintended scatter conditions and to position the detector parallel to the beam at the desired Source-to-Detector Distance (SDD). A cylindrical brass mini-phantom was fitted to the PSD to allow measurements beyond the depth of maximum dose. Irradiations were performed in a $3 \times 3 \mathrm{~cm}$ field using the maximum dose-rate available on the linac of 580 Monitor Units (MU) per minute. The SDD was varied from $70 \mathrm{~cm}$ to $130 \mathrm{~cm}$ and any deviation in doserate seen from that calculated by the inverse square law was recorded as dose-rate dependence. Measurements with $100 \mathrm{~cm}$ SDD setup were repeated three times throughout the experiment, interspersed with measurements at other SDDs, in order to assess the uncertainty associated with the positional accuracy.

The dose-per-pulse dependence of the detector was also evaluated by varying the linac dose rate setting from $100 \mathrm{MU} / \mathrm{min}$ up to $580 \mathrm{MU} /$ $\mathrm{min}$. For this test the detector was positioned at $5 \mathrm{~cm}$ depth in a WT1 phantom and irradiated with a $10 \times 10 \mathrm{~cm}$ field size at SSD $95 \mathrm{~cm}$ (SDD $100 \mathrm{~cm}$ ).

\subsection{Angular dependence}

The purpose of these tests was to check for dose dependence with change in incident angle of irradiation along both the symmetrical and polar axis of the PSD. The gantry angle was kept at $0^{\circ}$ for all measurements. The irradiations were performed with a $3 \times 3 \mathrm{~cm}$ field size in order to minimise Cherenkov emissions from the detector stem.

For the symmetrical axis angular dependence test, a cylindrical Perspex sleeve was drilled for the PSD and marked along its circumference at $0^{\circ}, 30^{\circ}, 90^{\circ}$ and $150^{\circ}$ rotations in the clockwise and anticlockwise directions. The detector was fixed inside the Perspex sleeve, which was then placed in a Perspex phantom. The sleeve was rotated using the indicated angle marks. The signal collected at each rotation was normalised to that at the reference angle $0^{\circ}$ and the dose deviations were recorded as angular dependence along the symmetrical axis.

For the polar axis angular dependence test, the PSD was initially positioned with its stem parallel to the beam in the IBA Blue water tank phantom (reference detector angle $0^{\circ}$ ). The alignment cap provided by the manufacturer was used to align the sensitive volume of the detector with the water surface and the central axis of the beam $(\mathrm{SSD}=95 \mathrm{~cm})$. In order to evaluate the effect of the beam profile shape on the long axis of the detector, measurements were performed at three different depths: $1.5 \mathrm{~cm}, 5 \mathrm{~cm}$ and $10 \mathrm{~cm}$. The PSD was rotated in $30^{\circ}$ steps from $0^{\circ}$ to $90^{\circ}$. The latter angle being where the PSD's stem becomes perpendicular to the beam axis. Measurements were performed in clockwise and anticlockwise directions. A goniometer was used to visually verify the angle of rotation. The signal collected at each angle was compared to the one at the reference angle $\left(0^{\circ}\right)$ and the deviations were recorded as angular dependence along the polar axis. The reference irradiation was repeated three times throughout the experiment, interspersed with measurements at other angles, in order to determine the positional uncertainty.

An additional test was performed, to evaluate the effect of the detector orientation on dose measurements. For this test, the detector was irradiated at a depth of $5 \mathrm{~cm}(95 \mathrm{~cm} \mathrm{SSD})$ in a $10 \times 10 \mathrm{~cm}$ field with a $6 \mathrm{MV}$ photon beam. The calibration was performed following the manufacturer's recommended method for irradiations in a water tank with the PSD's stem parallel to the beam axis (Morin et al., 2013). This calibration procedure requires measurements with approximately $10 \mathrm{~cm}$ of the fibre exposed for the minimum setup condition and 20$30 \mathrm{~cm}$ of fibre exposed for the maximum fibre setup condition. As recommended, the fibre bend radius was kept approximately the same for both conditions. The CLR and Gain (dose-to-water) factors from this calibration were then applied to measurements performed with the PSD's stem perpendicular to the beam axis. Additionally, calibration factors were acquired with the PSD's stem perpendicular to the beam axis, following the standard calibration procedure (Guillot et al., 2011). Similarly, these calibration factors were applied to measurements performed with the PSD's stem parallel to the beam axis. Five independent measurements were performed with both approaches and the mean readings were compared to the absolute dose measured with an NPL2611 ionisation chamber. The deviation seen from absolute dose was recorded as dose dependence to orientation-specific calibration factor determination.

\subsection{Temperature dependence}

A water bath, with a temperature calibration certificate traceable to the NPL, was used to maintain a temperature controlled environment for the PSD. The detector was secured in the water bath held by a Perspex stand, and held parallel to the beam at $5 \mathrm{~cm}$ depth $(95 \mathrm{~cm}$ SSD). The water bath was allowed to reach thermal equilibrium. Measurements where then performed with a thermistor placed close to the PSD. The temperature range investigated was from $18^{\circ} \mathrm{C}$ to $24{ }^{\circ} \mathrm{C}$ in order to account for the temperature variations likely to be met in different linac bunkers, during a multi-centre dosimetry audit. Standard room temperature $\left(20^{\circ} \mathrm{C}\right)$ was used as a reference for comparison of the detector response.

\subsection{Energy dependence}

It is known that PSD CLR factors are energy dependent (Dimitriadis et al., 2016b). The energy dependence was therefore investigated by calibrating the detector through a wide energy range (QI range of 0.568 for ${ }^{60} \mathrm{Co}$ up to 0.758 for $15 \mathrm{MV}$ ). Subsequently, measurements in all beams were performed and converted to dose, using the calibration factors determined for all the beam energies. The mean of five measurements was recorded for each combination of beam and calibration factors. Each of these combinations therefore produced a dose measurement. The measured doses were compared to the absolute dose measured using a calibrated PTW Semiflex ionisation chamber. Any deviations from the absolute dose were recorded as energy dependence. The whole procedure was repeated a second time and the average values from both sets of measurements were calculated. 


\subsection{Long-term stability}

The degradation of the scintillator response over its irradiation history needs to be evaluated. This is expressed as the loss of scintillation signal and is evident in the CLR factor change over accumulated dose. Studies with other PSDs suggest large losses of scintillation signal due to this effect (Taylor et al., 2011). However, it has recently been shown that acceptable levels of degradation can be achieved (Beaulieu et al., 2013). The manufacturer suggests that the detector should be recalibrated every $1 \mathrm{kGy}$ to account for this effect. The long term stability of the detector was evaluated by periodically repeating the CLR calibration process in the same $6 \mathrm{MV}$ beam over approximately $25 \mathrm{kGy}$ of irradiation history. The decrease of CLR in relation to the initial measured value, was recorded as the loss of sensitivity due to fibre degradation from accumulated dose.

\section{Results}

\subsection{Dose response, collection mode and short-term repeatability}

The measurements showed a linear response $\left(\mathrm{R}^{2}=1.000\right)$ in both pulsed and continuous radiation throughout the dose range investigated. There were no significant differences between triggered and manual collections for doses above $1 \mathrm{~Gy}$, where variations of up to \pm $0.1 \%$ were observed. For doses between 0.1 and $1 \mathrm{~Gy}$, manual collection readings were noticeably higher than triggered collection readings, with the differences reaching up to $2.5 \%$ for $0.1 \mathrm{~Gy}$. The detector's short-term stability was found to be within $\pm 0.3 \%$.

\subsection{Dose-rate and dose-per-pulse}

The deviation from the inverse square law in the dose-rate measurements was found to be within $\pm 0.5 \%$. Repeated measurements of the $100 \mathrm{~cm}$ SDD setup showed repeatability within $\pm 0.3 \%$, therefore indicating reasonable levels of setup accuracy. The dose-perpulse dependency was found to be slightly larger, reaching a maximum relative difference of $0.8 \%$ from the reference dose rate.

\subsection{Angular response}

The angular dependence along both axes of rotation of the PSD is shown in Fig. 2. The dependence along the symmetrical axis is very small, as expected, and does not exceed $0.3 \%$ from the response at the reference angle of $0^{\circ}$. Along the polar axis, larger differences were expected as the sensitive volume is cylindrical and its length is three times the size of its diameter. The largest differences were observed when the detector was at $5 \mathrm{~cm}$ deep and at $60^{\circ}-90^{\circ}$ rotation angles. The detector showed over-response when rotated from the parallel orientation $\left(0^{\circ}\right)$ towards the perpendicular orientation $\left(90^{\circ}\right)$. The dose dependence shown is close to $1 \%$ of the reference irradiation at angle $0^{\circ}$. Repeated measurements at angle $0^{\circ}$ showed a setup uncertainty of $\pm 0.3 \%$, similar to the detector's short-term stability, and therefore not indicative of additional sources of uncertainty.

When parallel-specific calibration factors were applied to perpendicular measurements, the detector over-responded by $0.5 \%$. When perpendicular-specific calibration factors were applied to parallel measurements, the detector under-responded by $0.7 \%$. This effect appears to be greater than the detector reproducibility and indicates that the dependence seen in the polar axis test, may be mainly attributed to calibration factor determination.

\subsection{Temperature dependence}

The temperature dependence of the PSD is shown in Fig. 3. The signal produced decreases at a rate of approximately $0.25 \%$ per ${ }^{\circ} \mathrm{C}$ throughout the temperature range investigated. Using the results
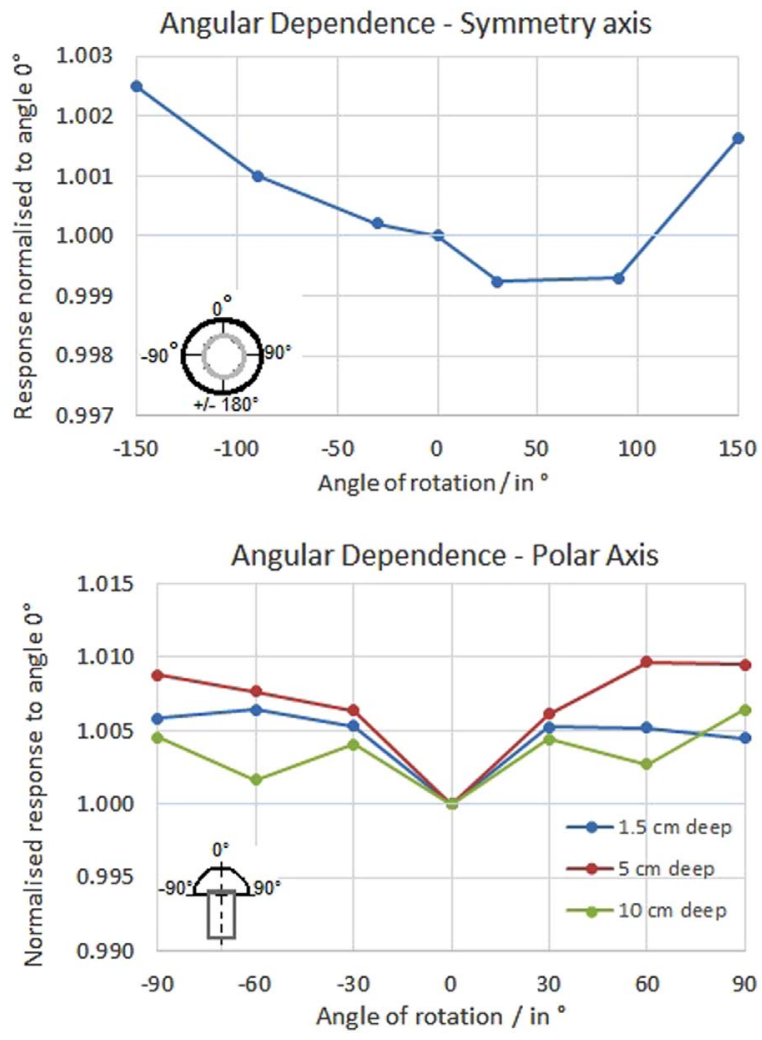

Fig. 2. Angular dependence of the Exradin W1 plastic scintillation detector.

Temperature dependence

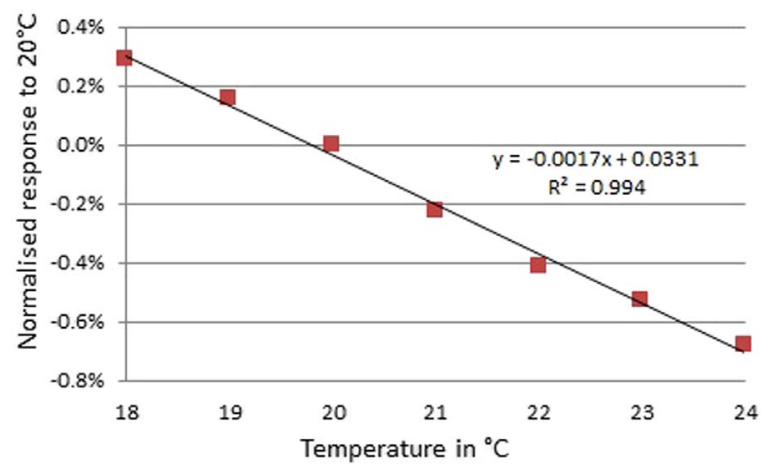

Fig. 3. Temperature dependence of the Exradin W1 plastic scintillation detector.

presented, it is possible to apply temperature corrections to the measurements performed during a dosimetry audit, in cases where a change in environmental conditions takes place.

\subsection{Energy dependence}

The results of the measurements performed with all calibration factors are shown in Table 1 . The deviations from absolute dose become noticeable when the calibration factors from a different beam quality are applied. These deviations become greater for greater energy differences between calibration factors and beam qualities. The maximum difference was seen when $15 \mathrm{MV}$ calibration factors were used in $\mathrm{a}^{60} \mathrm{Co}$ beam (1.99\%) and when ${ }^{60} \mathrm{Co}$ calibration factors were used in a 15 MV beam (-1.62\%). Reasonable agreement was seen with smaller differences in beam quality: $15 \mathrm{MV}$ factors used in a $10 \mathrm{MV}$ beam showed dose differences of $0.22 \%$. When the correct factors were used for all energies, the difference in absolute dose between the two 
Table 1

Deviations from absolute dose for each factor-energy combination with the Exradin W1. Underlined results show where the correct factors were used.

\begin{tabular}{lllll}
\hline Beam Energy & $\begin{array}{l}{ }^{60} \mathrm{Co}(\mathrm{QI}: \\
0.568)\end{array}$ & $\begin{array}{l}6 \mathrm{MV}(\mathrm{QI}: \\
0.682)\end{array}$ & $\begin{array}{l}10 \mathrm{MV}(\mathrm{QI}: \\
0.733)\end{array}$ & $\begin{array}{l}15 \mathrm{MV} \\
\text { (QI:0.758) }\end{array}$ \\
\hline$\frac{\text { Calibration }}{\underline{\text { factors }}}$ & & & & \\
${ }^{60} \mathrm{Co}$ & $\underline{0.04 \%}$ & $-0.83 \%$ & $-1.37 \%$ & $-1.62 \%$ \\
$6 \mathrm{MV}$ & $1.22 \%$ & $\underline{0.02 \%}$ & $-0.58 \%$ & $-0.99 \%$ \\
$10 \mathrm{MV}$ & $1.70 \%$ & $0.42 \%$ & $\underline{-0.01 \%}$ & $-0.55 \%$ \\
$15 \mathrm{MV}$ & $1.99 \%$ & $0.64 \%$ & $0.22 \%$ & $\underline{-0.04 \%}$ \\
\hline
\end{tabular}



Fig. 4. Long term stability of the Exradin W1 plastic scintillation detector.

detectors was within $0.04 \%$. This demonstrated that the Exradin W1 should be calibrated in the beam quality in which it is to be used.

\subsection{Long-term stability}

The loss of sensitivity of the PSD over $25 \mathrm{kGy}$ of exposures is plotted in Fig. 4. A rapid drop of approximately $8 \%$ was observed in the first 5 $\mathrm{kGy}$ delivered (1.6\% per $\mathrm{kGy}$ ). In the following $20 \mathrm{kGy}$ of exposures, the rate of loss of sensitivity decreased to approximately $0.2 \%$ per $\mathrm{kGy}$.

\section{Discussion}

The detector showed reproducibility within $0.3 \%$ in measurements, similar to the levels reported in previous studies (Beierholm et al., 2014; Carrasco et al., 2015a, 2015b). In order to achieve these levels of stability, the detector requires careful handling to ensure low leakage currents. Leakage appeared to become negligible when the photodiode box was shielded from scattered radiation and the optical fibre and cables were positioned with minimal strain applied to them. Since this system comprises sensitive electronics and produces signals that are many orders of magnitude smaller than other dosimetry systems, leakage currents can produce large errors in measurement. However, it was possible to achieve high levels of reproducibility using the methodology described.

Manual and triggered modes did not show significant differences in the collected readings for doses above $1 \mathrm{~Gy}$ but the differences increased by an order of magnitude for doses below $1 \mathrm{~Gy}$. The differences seen are likely related to the amount of phosphorescence (delayed luminescence) collected at the end of a reading. Triggered mode stops the collection as soon as the signal passes the predefined threshold whereas manual collection, at least using the method described, always had a longer acquisition time. As the dose becomes larger, the contribution from phosphorescence is proportionally smaller and becomes negligible above $1 \mathrm{~Gy}$. Carrasco et al. reported similar differences when using manual collection mode at low doses but they did not investigate doses above $1 \mathrm{~Gy}$. Also, the authors did not describe how the manual collection was acquired so it is possible that a different methodology was used. Using the method described in this study, it was possible to achieve agreement within $\pm 0.1 \%$ between the two collection modes. In SRS plan verification measurements, the detector is exposed to multiple radiation beams and/or heavily modulated beams where a large amount of the dose collected will be delivered from the penumbrae of different beams. The triggered mode is unreliable in ensuring appropriate start and stop time points for this type of collection, whereas the manual mode allows for this to be controlled by the user. The results demonstrated that using manual mode and the consistent collection method described, accurate measurements are acquired in the dose range of interest, making this method suitable for the proposed dosimetry audit.

The detector exhibits negligible angular dependence along the symmetrical axis, in agreement with published results (Carrasco et al., 2015a, 2015b). Along its polar axis, a dependence of approximately $1 \%$ was observed, which is relatively small in comparison to diamond detectors that exhibit dependencies of up to $3 \%$ (Ciancaglioni et al., 2012). This dependency has not been previously reported and is important to include within the uncertainty budget for non-coplanar deliveries, such as SRS. The determination of calibration factors, to be applied to SRS dose measurements, can only be performed using one of the two orientations. The standard perpendicular method is more reliable and practical for the purposes of a dosimetry audit. As shown in the results, a dose difference of up to $0.7 \%$ was observed when calibration factors were acquired at a different orientation from the measurement. It should be noted that the scenarios and uncertainties calculated from these tests are generous as the extreme conditions simulated are unlikely to be met in a clinical delivery. The tests accounted for situations where all of the dose is delivered from a single direction but in reality the delivery will occur over multiple directions and the effects shown should be significantly smaller.

The results show a spread of $1.8 \%$ in the CLR factor determination over the range of energies investigated. This was determined to be a maximum difference of $2 \%$ in the dose measured. This difference diminishes when the factors are from a similar beam quality and disappear when the factors are acquired at the same beam energy. As demonstrated, the detector has an energy dependence which can be eliminated by performing the calibration in the same beam where the measurements are going to be performed. It should be mentioned that the calibration process most practical for an audit requires irradiations in a $40 \times 40 \mathrm{~cm}$ field. These conditions are not possible for all SRS delivery machines. However, by acquiring calibration factors in a beam with a quality index as close as possible to the measurement beam, the dose difference observed becomes very small.

The results shown for the long term stability of the detector are comparable with published studies. Beierholm et al. show a rate of decrease in sensitivity of $2 \%$ per $\mathrm{kGy}$ over $1.5 \mathrm{kGy}$, comparable to the initial loss seen in this study (1.6\% per kGy). Carrasco et al. show the same trend in sensitivity loss but at different rates. However, the initial rate of loss seen in that study is similar to the latter rate seen in this study. The results presented are supportive to the speculations that such differences in detector characteristics could be related to different pre-irradiation exposures by the manufacturer, intended to overcome the need for frequent calibration (Carrasco et al., 2015a, 2015b). The results suggest that the detector's long term stability is sufficient for use in a multi-centre dosimetry audit. If a calibration is performed per $\mathrm{kGy}$ of exposures, the uncertainty contributed will be of the order of $0.2 \%$.

\subsection{Uncertainty budget}

The results presented in this study allowed for the calculation of an uncertainty budget for any measurements performed for dose verification in SRS. The analysis of uncertainty follows the JCGM Guide to the Expression of Uncertainty in Measurement (BIPM et al., 2008). 
Table 2

Uncertainty budget for the Exradin W1 plastic scintillation detector.

\begin{tabular}{llc}
\hline Source & \multicolumn{2}{l}{ Standard uncertainty (\%) } \\
\cline { 2 - 3 } & Type A & Type B \\
\hline Gain determination & \pm 1.2 & \\
Angular dependence & \pm 1.0 & \\
Dose rate dependence & \pm 0.8 & \\
CLR determination & \pm 0.7 & \pm 0.7 \\
Ion chamber measurement uncertainty & & \\
Setup accuracy & \pm 0.5 & \\
Long-term stability & \pm 0.2 & \\
Manual collection mode & \pm 0.1 & \\
Electrometer & & \\
Combined & & \\
\hline
\end{tabular}

Uncertainties evaluated by statistical analysis are grouped as type A and the remainder are grouped as type $\mathrm{B}$. These are added in quadrature to give a combined standard uncertainty with coverage factor $\mathrm{k}=1$. Table 2 shows the calculated uncertainty budget using the manual collection mode for doses above $1 \mathrm{~Gy}$, ranked from largest to smallest.

\section{Conclusions}

The Exradin W1 plastic scintillation detector was tested in various conditions to characterise its behaviour. The results show good agreement with published data and the dose dependencies to all parameters investigated are relatively small. A generous measurement uncertainty was calculated to account for the worst case scenario for measured doses above $1 \mathrm{~Gy}$.

This study has demonstrated that the PSD, when used in manual collection mode, with careful calibration and handling, constitutes an excellent dosimeter which can be employed in a multi-centre SRS dosimetry audit.

\section{References}

Beaulieu, L., Goulet, M., Archambault, L., Beddar, S., 2013. Current status of scintillation dosimetry for megavoltage beams. J. Phys. Conf. Ser. 444, 12013. http://dx.doi.org/ 10.1088/1742-6596/444/1/012013.

Beierholm, A.R., Behrens, C.F., Andersen, C.E., 2015. Comment on "characterization of the Exradin W1 scintillator for use in radiotherapy". Med. Phys. 42, 4414-4416. http://dx.doi.org/10.1118/1.4922656, (Med. Phys. 42, 297-304 )(2015)].

Beierholm, a.R., Behrens, C.F.F., Andersen, C.E.E., Beierholm, A.R., Behrens, C.F.F., Andersen, C.E.E., 2014. Dosimetric characterization of the Exradin W1 plastic scintillator detector through comparison with an in-house developed scintillator system. . Radiat. Meas. 69, 50-56. http://dx.doi.org/10.1016/ j.radmeas.2014.08.005.

BIPM, I.E.C., IFCC, I., ISO, I., IUPAP, O., 2008. Evaluation of measurement data guide to the expression of uncertainty in measurement, JCGM 100: 2008 GUM 1995 with minor corrections. Jt Comm. Guid. Metrol.

Carrasco, P., Jornet, N., Jordi, O., Lizondo, M., Eudaldo, T., Ruiz, A., Ribas, M., Carrasco, P., Jornet, N., Jordi, O., Lizondo, M., Eudaldo, T., Ruiz, A., 2015a. Characterization of the Exradin W1 scintillator for use in radiotherapy Characterization of the Exradin W1 scintillator for use in radiotherapy. Med. Phys. 297, 297-304. http://dx.doi.org/ 10.1118/1.4903757.

Carrasco, P., Jornet, N., Jordi, O., Lizondo, M., Latorre-Musoll, A., Eudaldo, T., Ruiz, A Ribas, M., 2015b. Response to "comment on "characterization of the Exradin W1 scintillator for use in radiotherapy"'. Med. Phys. 42, 4417-4418. http://dx.doi.org/ 10.1118/1.4922655, (Med. Phys. 42, 297-304 )(2015)].

Ciancaglioni, I., Marinelli, M., Milani, E., Prestopino, G., Verona, C., Verona-Rinati, G., Consorti, R., Petrucci, A., De Notaristefani, F., 2012. Dosimetric characterization of a synthetic single crystal diamond detector in clinical radiation therapy small photon beams. Med. Phys. 39, 4493-4501. http://dx.doi.org/10.1118/1.4729739.

Dimitriadis, A., Archambault, L., Clark, C.H., Bouchard, H., 2016a. Modelling the energy dependence of Cherenkov light in plastic scintillation detectors. Radiother. Oncol. 119, 716-717. http://dx.doi.org/10.3252/pso.eu.ESTRO2016.2016.

Dimitriadis, A., Kirkby, K.J., Nisbet, A., Clark, C.H., 2016b. Current status of cranial stereotactic radiosurgery in the UK. Br. J. Radiol. 89, 20150452. http://dx.doi.org/ 10.1259/bjr.20150452.

Guillot, M., Gingras, L., Archambault, L., Beddar, S., Beaulieu, L., 2011. Spectral method for the correction of the Cerenkov light effect in plastic scintillation detectors: a comparison study of calibration procedures and validation in Cerenkov lightdominated situations. Med. Phys. 38, 2140-2150. http://dx.doi.org/10.1118/ 1.3562896.

Kamio, Y., Bouchard, H., 2014. Correction-less dosimetry of nonstandard photon fields: a new criterion to determine the usability of radiation detectors. Phys. Med. Biol., 59.

Klein, D., Briere, T.M., Kudchadker, R., Archambault, L., Beaulieu, L., Lee, A., Beddar, S. 2012. In-phantom dose verification of prostate IMRT and VMAT deliveries using plastic scintillation detectors. Radiat. Meas. 47, 921-929. http://dx.doi.org/ 10.1016/j.radmeas.2012.08.005.

Liu, P.Z.Y., Suchowerska, N., Lambert, J., Abolfathi, P., McKenzie, D.R., 2012. Reply to the comment on: "plastic scintillation dosimetry: comparison of three solutions for the Cerenkov challenge. Med. Phys. 57, 3667-3673. http://dx.doi.org/10.1088/ 0031-9155/57/11/3667.

Morin, J., Beliveau-Nadeau, D., Chung, E., Seuntjens, J., Theriault, D., Archambault, L., Beddar, S., Beaulieu, L., 2013. A comparative study of small field total scatter factors and dose profiles using plastic scintillation detectors and other stereotactic dosimeters: the case of the CyberKnife. Med. Phys. 40, 11719. http://dx.doi.org/ $10.1118 / 1.4772190$

Ottosson, W., Behrens, C.F., Andersen, C.E., 2015. Dose verification of radiotherapy for lung cancer by using plastic scintillator dosimetry and a heterogeneous phantom. J. Phys. Conf. Ser. 573, 12022. http://dx.doi.org/10.1088/1742-6596/573/1/012022.

Pasquino, M., Stasi, M., Mancosu, P., Russo, S., Villaggi, E., Gasperi, C., Casale, M, ., Loi, G., Strigari, L., Miceli, R., Raza, G.H., Fedele, D., Vaiano, A., Falco, M.D., Moretti, E., Giglioli, F.R., Nigro, R., Talamonti, C., Pastore, G., Luxardo, S., Menghi, E., Benecchi, G., Clemente, S., Marino, C., Borzi, G., Nardiello, B., Ardu, V., Paladini, L., Cagni, E., Russo, G., Spiazzi, L., Vittorini, F., 2016. Dosimetric characterization of linac small beams using a plastic scintillator detector: A multicenter study, in: 1st European Congress of Medical Physics. Athens, pp. 225-226. http://dx.doi.org/10.1016/j. ejmp.2016.07.466.

Silvestre, I., Dimitriadis, A., Subiel, A., 2016. Relative output factors for TPS beam data acquisition with emphasis on small fields. Comparison of multiple detectors and multiple approaches, In: Proceedings of the ALFIM, 7th Latin American Conference in Medical Physics. Cordoba, Argentina.

Taylor, M.L., Kron, T., Franich, R.D., 2011. A contemporary review of stereotactic radiotherapy: inherent dosimetric complexities and the potential for detriment. Acta Oncol. 50, 483-508. http://dx.doi.org/10.3109/0284186X.2010.551665.

Underwood, T.S., Winter, H.C., Hill, M.A., Fenwick, J.D., 2013. Detector density and small field dosimetry: integral versus point dose measurement schemes. Med. Phys. 40. http://dx.doi.org/10.1118/1.4812687. 